\title{
Lipid-induced cell stress and insulin
} resistance

\author{
Patrick Schrauwen', Joris Hoeks' and Matthijs KC Hesselink ${ }^{2}$ \\ Department of 'Human Biology; ${ }^{2}$ Movement Sciences, Maastricht University, Wageningen Center for Food Sciences \\ (WCFS) and Nutrition and Toxicology Research Institute Maastricht (NUTRIM), Maastricht, The Netherlands
}

\begin{abstract}
In our Westernized society, although some excess body fat is stored inside its proper place, adipose tissue, the surplus of circulating fatty acids is also excessively stored in the liver, heart, pancreas and skeletal muscle. In these tissues, intracellular fat accumulation, in combination with a low oxidative capacity, is associated with decreased insulin sensitivity. Although the exact mechanism behind the negative effect of intracellular lipid accumulation on insulin sensitivity has not been completely unravelled, ample evidence suggests that fatty acid intermediates, such as diacylglycerol, fatty acyl-coenzyme-A and ceramides, can hamper insulin signalling. However, recent evidence also points towards a role for reactive oxygen species in the development of insulin resistance. The present review will focus on how fatty acids in combination with oxidative stress can lead to lipid-induced oxidative damage, thereby impairing mitochondrial function and facilitating the accumulation of muscular lipids.
\end{abstract}

Keywords: type 2 diabetes; muscle; mitocondria; UCP3; reactive oxygen species; fatty acid

Abbreviations: ATP: adenosine triphosphate; CPT: carnitine palmitoyl-transferase; DAG: diacylglycerol; FFA: free fatty acid; IMCL: intramyocellular lipid; NADH: nicotinamide adenine dinucleotide; PKC: protein kinase $C$; ROS: reactive oxygen species; TNF: tumour necrosis factor; UCP: uncoupling protein.

\section{Introduction}

$\mathrm{O}$ ver the past few decades, the incidence of type 2 diabetes mellitus has increased dramatically. In 2003, about 194 million people, equalling $5.1 \%$ of the global population in the age group 20-79 years, were estimated to have diabetes (1). It is estimated that the worldwide number of people suffering from type 2 diabetes will approximate 330 million in 2025, with almost $10 \%$ (472 million) of the global population suffering from impaired glucose tolerance (1). The continuous increase in type 2 diabetes prevalence contributes significantly to illness and mortality rates.

At the heart of the aetiology of type 2 diabetes lies insulin resistance, an early detectable characteristic preceding the development of full-blown and clinically overt type 2 diabetes. Insulin resistance indicates a state of reduced responsiveness of the insulin sensitive tissues to circulatory levels of insulin, i.e. reduced glucose disposal and impaired inhibition of hepatic glucose output at a given concentration of insulin (2). A major contribution to whole body insulin resistance comes from the skeletal muscle, as revealed by classic work by
DeFronzo et al. (3). They showed a $45 \%$ reduction in leg glucose uptake following a hyperinsulinaemic euglycaemic clamp in type 2 diabetic patients, and a strong positive correlation $(r=0.70, p<0.001)$ between leg and total body glucose uptake (3).

Not only are plasma glucose levels increased in uncontrolled type 2 diabetes, but also increased plasma free fatty acids (FFAs) and increased storage of fatty acids in tissues including nonadipose tissue such as muscle, pancreas and liver have long been recognized (4). In 1992, McGarry (2) described the importance of fatty acids and lipid metabolism in the development of type 2 diabetes mellitus, suggesting that insulin resistance and concomitant hyperglycaemia would be easier to understand if viewed in the context of underlying abnormalities of lipid metabolism. With the lipocentric view in mind, the term lipotoxicity was first used by Unger, referring to the deteriorating effect of triglyceride accumulation in pancreatic $\beta$-cells on glucose-stimulated insulin secretion (5). Later, the concept of lipotoxicity was extended towards skeletal muscle, where it was linked directly to insulin resistance (6). Skeletal muscle is able to store lipid 
droplets within the myocyte; these intramyocellular lipids (IMCLs) are located in the near vicinity of mitochondria, suggesting that IMCLs can serve as a rapidly available energy source. However, despite having a physiological function in providing energy for the mitochondria, IMCLs appear to have detrimental effects on muscular insulin sensitivity, as they have been shown to correlate strongly with insulin resistance (7). Remarkably, the amount of IMCL is very sensitive to diet, as it has been shown that the consumption of a high-fat diet for 7 days leads to an increase in IMCL stores by approximately $50 \%$ in lean, healthy subjects (8). In addition, levels of IMCL are increased in insulinresistant, but non-diabetic first degree relatives of type 2 diabetic patients, suggesting that IMCL accumulation may be primary in the development of type 2 diabetes (9). Combined, these data suggest that the increase in IMCLs on a high-fat diet may lead to the development of muscular insulin resistance. Although no direct evidence is available that the high-fat diet-induced increase in IMCLs in humans predisposes to the development of insulin resistance, rodent studies did reveal such causal relationship. Thus, Dobbins et al. (10) showed that the dietary effects on muscular triglyceride content correlated strongly with the development of muscular insulin resistance. Despite these findings, a causal relation between IMCL content and insulin resistance has been strongly questioned because of the paradoxical finding that endurance-trained athletes, who are among the most insulin-sensitive subjects, also have increased IMCL content (11), and that IMCL content is rapidly increased after the onset of an endurance training programme (12). At present, this paradox is explained by other fatty acid intermediates affecting insulin sensitivity: in the insulin-resistant state IMCLs may just function as a marker of these fatty acid intermediates, which accumulate if not all fatty acids that are released from intracellular triglycerides can be (efficiently) directed towards oxidation. Thus, in a condition of excessive fatty acid supply and low oxidative capacity (as with a high-fat diet and diabetes), both IMCLs and the fatty acid intermediates accumulate and the latter impair insulin signalling. In contrast, with endurance training, characterized by high oxidative capacity, fatty acid intermediates are expected to stay low despite high IMCL levels, and the high IMCL levels serve to provide the regularly exercising muscle with abundant energy stores.

One of the fatty acid intermediates that may specifically be linked to the induction of insulin resistance is diacylglycerol. Diacylglycerol can activate distinct isoforms of protein kinase $\mathrm{C}$ (PKC) and activated PKC can directly impede insulin signalling by phosphorylating and inhibiting tyrosine kinase activity of the insulin receptor and tyrosine phosphorylation of insulin receptor substrate-1, which results in inactivation of the insulin receptor (for review see ref. 13). Much of the evidence for this concept comes from studies in which the acute effect of increased FFA levels on insulin sensitivity has been examined. Infusion of Intralipid ${ }^{\circledR}$ to increase FFA levels during a hyperinsulinaemic euglycaemic clamp reduces insulinmediated glucose uptake within 2-4 h of fat infusion, which is paralleled by increased levels of diacylglycerol (DAG) and PKC activation (14). The importance of DAG-induced activation of PKC follows from studies with mice lacking PKC, which are protected from the development of lipidinduced insulin resistance (15), providing further evidence for an important role of this pathway in the development of muscular insulin resistance. Therefore, preventing DAG accumulation and/or PKC activation may be a therapeutic strategy to overcome insulin resistance and the development of type 2 diabetes. In that context, it has been shown that DAG accumulation and PKC activation are differentially affected by different types of fatty acid: whereas saturated fatty acids led to DAG accumulation and PKC activation in a cellular model, this effect was not observed in comparable concentrations of unsaturated fatty acids (16). Although more research is needed, this finding may provide a mechanistic explanation for the observation that diets with unsaturated fatty acids can have a beneficial effect on the development of type 2 diabetes mellitus (17).

\section{A role for oxidative stress in the development of insulin resistance}

Although the accumulation of fatty acid intermediates in muscle (and also liver and heart) is nowadays widely accepted as being linked to the development of type 2 diabetes, research in this field has recently also pointed towards the role of oxidative stress in the development of this disease. In a very recent paper, Houstis et al. (18) provided evidence for a 
causal role for reactive oxygen species (ROS) in the development of insulin resistance. They found that cellular ROS levels were elevated in two cell models of insulin resistance, where insulin resistance is induced by treatment with either the cytokine tumour necrosis factor- $\alpha$ (TNF- $\alpha)$ or the glucocorticoid dexamethasone. To provide evidence for a causal relation between the elevated ROS levels and insulin resistance, the authors overexpressed a variety of antioxidant genes in their model, and the overexpression of each of these genes could reverse the insulin resistance that was induced by both TNF- $\alpha$ and dexamethasone (18). The question then arise as to whether ROS production is elevated in (pre)-diabetic subjects and how oxidative stress can lead to (muscular) insulin resistance.

The major contributor to ROS production are the mitochondria, and mitochondrial ROS can react rapidly with DNA, protein and lipids, thereby leading to oxidative damage. Fatty acids are especially prone to oxidative damage, resulting in the formation of lipid peroxides, which in turn can induce damage to proteins and DNA. Therefore, accumulation of fatty acids in the vicinity of the mitochondrial matrix, where ROS are formed, increases the likelihood of lipid peroxidation. As discussed above, type 2 diabetic patients are characterized by the accumulation of IMCL and these lipid droplets are located close to the mitochondria. To prevent fatty acids simply diffusing into the mitochondria, the entry of fatty acids into the mitochondria is regulated by the enzyme carnitine palmitoyl-transferase (CPT-1), which serves as an inward transporter of oxidizable fatty acids. However, this system cannot completely prevent the diffusion of fatty acid into the mitochondria, as the mitochondrial membranes consist of lipid bilayers and fatty acids can still enter the mitochondria matrix via a "flip-flop" mechanism over the membrane (19). This passive diffusion may be more likely to occur under conditions of high intramyocellular lipid concentration, as is the case for insulin resistance and type 2 diabetic mellitus. Consistent with this idea, the skeletal muscle of obese insulinresistant subjects not only contains a higher amount of intramyocellular lipid, but also shows a higher degree of lipid peroxidation (20). Potentially, these lipid peroxides could lead to oxidative damage to mitochondrial structures. Indeed, it has been shown mitochondria from type 2 diabetic patients are smaller and show more mitochondrial damage, and that the mitochondrial area correlates positively with insulin sensitivity (21). This also results in an impaired functional capacity of mitochondria in skeletal muscle of diabetic patients (21). Thus, mitochondria of type 2 diabetic patients have a reduced capacity of the electron transport chain, as measured by nicotinamide adenine dinucleotide $(\mathrm{NADH}): \mathrm{O}_{2}$ oxidoreductase activity and reduced citrate synthase activity. In vivo, the rate of mitochondrial oxidative-phosphorylation activity, measured by $\left[{ }^{31} \mathrm{P}\right]$ magnetic resonance spectroscopy, was found to be reduced in skeletal muscle of insulinresistant offspring of type 2 diabetic patients (22). These studies together show that mitochondrial capacity is reduced in type 2 diabetic patients, which may be attributed to lipid-induced oxidative stress. In turn, this reduced mitochondrial capacity would favour the accumulation of fatty acid intermediates such as diacylglycerol, which may explain how lipidinduced oxidative stress may ultimately lead to (muscular) insulin resistance.

\section{Mitochondrial uncoupling as a mechanism to reduce lipid peroxidation}

If mitochondrial ROS production can be harmful to its own structure, the question arises how mitochondria are able to limit the production of ROS. It has been established that the production of superoxide (the primary ROS produced) is strongly related to the mitochondrial membrane potential, because of increased non-specific single-electron reductions of molecular oxygen at complexes I and III (23). Important work in this field has been performed by Skulachev (24), who showed that mild uncoupling may be effective in lowering mitochondrial ROS production. Mild uncoupling implies a limited increase in proton conductance of the inner mitochondrial membrane so that the membrane potential is slightly lowered, reducing the production of ROS without major effects on adenosine triphosphate (ATP) production. In 1997, the relevance of controlled mild uncoupling was extended to muscle physiology by the discovery of an uncoupling protein (called UCP3) that is present in skeletal muscle mitochondria. Shortly after its discovery it was suggested that UCP3 may contribute to the reduction of ROS by mediating mild uncoupling. The regulation of UCP3 content is strongly associated with fatty acid metabolism: UCP3 is upregulated or highly expressed when the supply of fatty acid to muscle exceeds the oxidative capacity 
(lipid infusion, high-fat diets, glycolytic muscle), and is low or down-regulated when the oxidative capacity is high (endurance training, oxidative muscle). This pattern of regulation is consistent with a protective role for UCP3 in preventing lipid-induced oxidative damage, as UCP3 is induced in conditions in which the chance of lipid-induced oxidative damage is high. Because UCP3 is able to export fatty acid anions and/or peroxides from mitochondria, it may indeed protect specifically against lipid-induced oxidative damage. It exerts its protective function in a dual manner, by exporting non-metabolizable fatty acids/peroxides that enter the mitochondria via passive diffusion before the lipid peroxide products can damage the mitochondrial machinery present in the mitochondrial matrix, and by exporting these negatively charged fatty acid substrates, thus lowering the proton gradient and limiting ROS production.

It was shown recently that UCP3 is activated by 4-hydroxy-2-nonenal, an important by-product of lipid peroxidation (25), suggesting a negative feedback loop involved in the regulation of mitochondrial lipid peroxide production. Evidence for a role for UCP3 in the protection of lipid-induced oxidative damage comes from experiments using mice lacking UCP3; these mice are characterized by increased levels of lipid peroxides (26) and damage to mitochondrial proteins (27). Conversely, overexpression of UCP3 in a cellular model was associated with reduced ROS production (28).

\section{Uncoupling protein-3 and type 2 diabetes mellitus}

The increased accumulation of muscular fat, the higher degree of lipid peroxidation and mitochondrial damage observed in insulin-resistant and/or type 2 diabetic patients could be linked if the abovementioned negative feedback loop were disturbed in type 2 diabetic patients. Indeed, type 2 diabetic patients and subjects with impaired glucose tolerance, often considered "prediabetics", have a 50\% reduction in UCP3 levels (29). These data suggest that in patients with type 2 diabetes the reduction in UCP3 is a pathological condition, in which low levels of UCP3 fail to protect mitochondria sufficiently against lipid-induced mitochondrial damage, indicating a defective feedback mechanism between lipid peroxides and mitochondrial uncoupling. This idea is further supported by the finding that in prediabetic subjects (with impaired glucose tolerance), a 1-year lifestyle diabetes prevention programme comprising increased physical activity, which effectively resulted in improved metabolic control, resulted in a two-fold increase in UCP3, thereby restoring UCP3 to normal physiological levels (30). In addition, in type 2 diabetic patients UCP 3 content was restored after a 1 year endurance training programme in parallel with improvement in insulin sensitivity (30). Finally, the novel antidiabetic agent Rosiglitazone up-regulates the protein content of UCP3 in skeletal muscle of type 2 diabetic patients, accompanied with an improved oxidative capacity, complex 2 activity and muscular insulin sensitivity (29). These findings in humans do not prove that the reduced levels of UCP3 are causally related to the development of lipid-induced oxidative damage and insulin resistance, and further studies are needed to prove a functional role for UCP3 in type 2 diabetes. Nevertheless, this suggestion is also supported by the recent finding that genetic variation in the UCP3 gene was associated with the 10- and 15-year risk of developing type 2 diabetes mellitus (31).

\section{Conclusion}

Patients with type 2 diabetes are characterized by low fat oxidative capacity and high plasma FFA levels, leading to accumulation of fatty acids inside muscle cells. Fat accumulation inside muscle cells will lead to the entry of fatty acids into mitochondria, where these fatty acids are prone to peroxidation. In turn, these lipid peroxides may damage mitochondria and further deteriorate oxidative capacity. Diabetic patients have increased mitochondrial damage and impaired mitochondrial function. UCP3 plays an important role in the protection of mitochondria against these lipid peroxides, by exporting fatty acid anions and/or peroxides from the mitochondria, and lowering ROS production. This function of UCP3 is activated by lipid peroxides, suggesting a negative feedback loop limiting lipid-induced oxidative damage. It is of note, however, that in patients with type 2 diabetes mellitus UCP3 levels are decreased by $50 \%$ compared with controls matched for age and body mass index, suggesting a defect in this negative feedback loop, which ultimately may lead to lipid-induced mitochondrial damage and insulin resistance. 


\section{References}

1. Dahlquist IG, Patterson C, Shaw J, Sicree R, Singh R, Soltesz G, et al. Diabetes e-atlas; 2005.

2. McGarry JD. What if Minkowski had been ageusic? An alternative angle on diabetes. Science 1992; 258: 766-70.

3. DeFronzo RA, Gunnarsson R, Bjorkman O, Olsson M, Wahren J. Effects of insulin on peripheral and splanchnic glucose metabolism in noninsulin-dependent (type II) diabetes mellitus. J Clin Invest 1985; 76: 149-55.

4. Schalch DS, Kipnis DM. Abnormalities in carbohydrate tolerance associated with elevated plasma nonesterified fatty acids. J Clin Invest 1965; 44: 2010-20.

5. Unger RH. Lipotoxicity in the pathogenesis of obesitydependent NIDDM. Genetic and clinical implications. Diabetes 1995; 44: 863-70.

6. Unger RH, Orci L. Lipotoxic diseases of nonadipose tissues in obesity. Int J Obes Relat Metab Disord 2000; 24(Suppl 4): S28-32.

7. Perseghin G, Scifo P, De Cobelli F, Pagliato E, Battezzati A, Arcelloni C, et al. Intramyocellular triglyceride content is a determinant of in vivo insulin resistance in humans: a ${ }^{1} \mathrm{H}-{ }^{13} \mathrm{C}$ nuclear magnetic resonance spectroscopy assessment in offspring of type 2 diabetic parents. Diabetes 1999; 48: 1600-6.

8. Schrauwen-Hinderling VB, Kooi ME, Hesselink MK, Moonen-Kornips E, Schaart G, Mustard KJ, et al. Intramyocellular lipid content and molecular adaptations in response to a 1-week high-fat diet. Obes Res 2005; 13: 2088-94.

9. Jacob S, Machann J, Rett K, Brechtel K, Volk A, Renn $\mathrm{W}$, et al. Association of increased intramyocellular lipid content with insulin resistance in lean nondiabetic offspring of type 2 diabetic subjects. Diabetes 1999; 48: $1113-9$.

10. Dobbins RL, Szczepaniak LS, Bentley B, Esser V, Myhill J, McGarry JD. Prolonged inhibition of muscle carnitine palmitoyltransferase-1 promotes intramyocellular lipid accumulation and insulin resistance in rats. Diabetes 2001; 50: 123-30.

11. Goodpaster BH, He J, Watkins S, Kelley DE. Skeletal muscle lipid content and insulin resistance: evidence for a paradox in endurance-trained athletes. J Clin Endocrinol Metab 2001; 86: 5755-61.

12. Schrauwen-Hinderling VB, Schrauwen P, Hesselink MK, Van Engelshoven JM, Nicolay K, Saris WH, et al. The increase in intramyocellular lipid content is a very early response to training. J Clin Endocrinol Metab 2003; 88: 1610-6.

13. Kelley DE, Mandarino LJ. Fuel selection in human skeletal muscle in insulin resistance: a reexamination. Diabetes 2000; 49: 677-83.

14. Itani SI, Ruderman NB, Schmieder F, Boden G. Lipidinduced insulin resistance in human muscle is associated with changes in diacylglycerol, protein kinase $\mathrm{C}$, and IkappaB-alpha. Diabetes 2002; 51: 2005-11.

15. Kim JK, Fillmore JJ, Sunshine MJ, Albrecht B, Higashimori T, Kim DW, et al. PKC-theta knockout mice are protected from fat-induced insulin resistance. J Clin Invest 2004; 114: 823-7.
16. Chavez JA, Summers SA. Characterizing the effects of saturated fatty acids on insulin signaling and ceramide and diacylglycerol accumulation in 3T3-L1 adipocytes and C2C12 myotubes. Arch Biochem Biophys 2003; 419: 101-9.

17. Vessby B, Unsitupa M, Hermansen K, Riccardi G, Rivellese AA, Tapsell LC, et al. Substituting dietary saturated for monounsaturated fat impairs insulin sensitivity in healthy men and women: the KANWU study. Diabetologia 2001; 44: 312-9.

18. Houstis N, Rosen ED, Lander ES. Reactive oxygen species have a causal role in multiple forms of insulin resistance. Nature 2006; 440: 944-8.

19. Ho JK, Duclos RI Jr, Hamilton JA. Interactions of acyl carnitines with model membranes: a ${ }^{13} \mathrm{C}-\mathrm{NMR}$ study. J Lipid Res 2002; 43: 1429-39.

20. Russell AP, Gastaldi G, Bobbioni-Harsch E, Arboit P, Gobelet C, Deriaz O, et al. Lipid peroxidation in skeletal muscle of obese as compared to endurance-trained humans: a case of good vs. bad lipids? FEBS Lett 2003; 551: 104-6.

21. Kelley DE, He J, Menshikova EV, Ritov VB. Dysfunction of mitochondria in human skeletal muscle in type 2 diabetes. Diabetes 2002; 51: 2944-50.

22. Petersen KF, Dufour S, Befroy D, Garcia R, Shulman GI. Impaired mitochondrial activity in the insulinresistant offspring of patients with type 2 diabetes. $\mathrm{N}$ Engl J Med 2004; 350: 664-71.

23. Turrens JF. Superoxide production by the mitochondrial respiratory chain. Biosci Rep 1997; 17: 3-8.

24. Skulachev VP. Role of uncoupled and non-coupled oxidations in maintenance of safely low levels of oxygen and its one-electron reductants. Q Rev Biophys 1996; 29: 169-202.

25. Echtay KS, Esteves TC, Pakay JL, Jekabsons MB, Lambert AJ, Portero-Otin M, et al. A signalling role for 4-hydroxy-2-nonenal in regulation of mitochondrial uncoupling. EMBO J 2003; 22: 4103-10.

26. Hoeks J, Hesselink MK, Sluiter W, Schaart G, Willems J, Morrisson A, et al. The effect of high-fat feeding on intramuscular lipid and lipid peroxidation levels in UCP3-ablated mice. FEBS Lett 2006; 580: 1371-5.

27. Brand MD, Pamplona R, Portero-Otin M, Requena JR, Roebuck SJ, Buckingham JA, et al. Oxidative damage and phospholipid fatty acyl composition in skeletal muscle mitochondria from mice underexpressing or overexpressing uncoupling protein 3. Biochem J 2002; 368: 597-603.

28. MacLellan JD, Gerrits MF, Gowing A, Smith PJ, Wheeler MB, Harper ME. Physiological increases in uncoupling protein 3 augment fatty acid oxidation and decrease reactive oxygen species production without uncoupling respiration in muscle cells. Diabetes 2005; 54: 2343-50.

29. Schrauwen P, Mensink M, Schaart G, Moonen-Kornips E, Sels JP, Blaak EE, et al. Reduced skeletal muscle uncoupling protein- 3 content in prediabetic subjects and type 2 diabetic patients: restoration by rosiglitazone treatment. J Clin Endocrinol Metab 2006; 91: 1520-5. 
30. Mensink M, Hesselink MK, Borghouts LB, Keizer H, Moonen-Kornips E, Schaart G, et al. skeletal muscle uncoupling protein-3 restores upon intervention in the pre-diabetic and diabetic state: implications for diabetes pathogenesis? Diabetes Obes Metab 2006; in press.

31. Gable DR, Stephens JW, Cooper JA, Miller GJ, Humphries SE. Variation in the UCP2-UCP3 gene cluster predicts the development of type 2 diabetes in healthy middle-aged men. Diabetes 2006; 55: 1504-11.

\section{Patrick Schrauwen}

Wageningen Center for Food Sciences "(WCFS)" and Nutrition and Toxicology Research Institute Maastricht (NUTRIM)

Maastricht University

PO Box 616

NL-6200 MD Maastricht

The Netherlands

Tel: +3I 43 388I502

Fax: +31 433670976

E-mail: p.schrauwen@hb.unimaas.nl 